\title{
目
}

\section{THE USE OF MEDIUM-CHAIN FATTY ACIDS IN PIGLET FEEDING - A REVIEW}

\author{
Ewa Hanczakowska
}

\begin{abstract}
Department of Animal Nutrition and Feed Science, National Research Institute of Animal Production, 32-083 Balice n. Kraków, Poland

Corresponding author: ewa.hanczakowska@izoo.krakow.pl
\end{abstract}

\begin{abstract}
The group of medium-chain fatty acids (MCFA) comprises monocarboxylic fatty acids containing from 6 to 12 carbon atoms. These are: caproic (C6), caprylic (C8), capric (C10), and lauric (C12) acids. They can be partly absorbed already through the stomach mucosa. Their triacylglycerols (MCT) can be absorbed intact into intestinal epithelial enterocytes and then hydrolysed by microsomal lipases. Thus they are a readily available source of energy, capable of improving the intestinal epithelial mucosal structure. They are also characterised by strong antibacterial activity due to their ability to penetrate the semi-permeable membranes of bacteria and damage their internal structures. Thanks to these properties, they could be a good supplement to weaned piglet feed. They improve piglet performance and can be used as feed antibiotic replacers.
\end{abstract}

Key words: medium-chain fatty acids, antibacterial activity, piglet nutrition

Acids, mainly organic, have been used for many years for the preservation of feed for pigs and other farm animals. In early experiments, propionic acid $(1.5 \%)$ was used for the preservation of wet corn used in diets for growing pigs (Young et al., 1970). There were no problems with mould growth during storage, and pigs fed experimental feed gained at a similar rate and achieved a feed efficiency equal to or better than that of pigs fed dry corn. Also, Bayley et al. (1974) found that nitrogen was better utilised in diets preserved with organic acids (propionic and acetic) than in dried-corn feed. A higher level of free glucose, resulting from partial hydrolysis of starch, was found in the acid-preserved corn. In contrast to results with older pigs, supplements of propionic acid lowered the daily gains of piglets, though fumaric and citric acids yielded better results (Giesting and Easter, 1985). In a later experiment, no effects of these last two acids on the retention of amino acids were found in piglets (Walz and Pallauf, 1997). 
The effects of organic acids frequently fed to pigs have recently been reviewed by Suiryanrayna and Ramana (2015). Another organic acid with a positive effect on piglet performance is butyric acid (Lu et al., 2008), a short-chain fatty acid with four carbon atoms in the chain and a good source of energy for intestinal epithelial cells. Piva et al. (2002) found that adding a butyrate supplement to piglet feed already acidified with formic and lactic acids improved body weight gains by about $20 \%$.

Several years ago, the European Union banned the use of antibiotic growth promoters in farm animal feeding (Anadón, 2006); thus some replacers must be found, especially for young animals. Various agents to prevent piglet gastrointestinal disorders have been proposed, among them short-chain fatty acids, used for many years as antimicrobial acidifiers (Partanen and Mroz, 1999). Medium-chain fatty acids (MCFA) constitute another type of organic acid which could be considered for use as antibiotic replacers (Decuypere and Dierick, 2003). They are characterised by strong antibacterial activity (Skrivanová et al., 2009) and, in addition to this antimicrobial effect, can also improve postweaning gut development (Loh et al., 2013).

In 2003 Decuypere and Dierick published a thorough overview of experiments with MCFAs. The aim of the present review is to present new experiments on the properties of MCFA and their triacylglycerols and to evaluate their effects on the health and performance of pigs, especially of weaned piglets.

\section{The effect of MCFAs on piglet performance}

The group MCFA comprises monocarboxylic fatty acids containing from 6 to 12 carbon atoms. The 'normal' (i.e. with an even number of carbon atoms) acids are caproic (C6), caprylic (C8), capric (C10), and lauric (C12) acids. Their systematic names are, respectively: hexanoic, octanoic, decanoic, and dodecanoic acids. Because pure medium-chain fatty acids have an unpleasant smell (Oprean et al., 2011), their triacylglycerols (MCT) are usually used in animal feeding. In the animal digestive tract MCT are preferentially hydrolysed by preduodenal lipases; thus the resulting MCFA can be partly absorbed already through the stomach mucosa. Moreover, MCT can be absorbed (intact) into intestinal epithelial enterocytes and then hydrolysed in cells by microsomal lipases (Playoust and Isselbacher, 1964). Portion of resulting MCFA entry into mitochondria is probably independent of the carnitine acyltransferase system (Odle, 1997). Also their oxidation should be carnitine independent because they are activated within the mitochondrial matrix (Heo et al., 2002). According to Loh et al. (2013), MCT have a lesser tendency to be stored as body fat and their rapid transportation and oxidation is more similar to carbohydrates than to other fats.

In an experiment by Guillot et al. (1994), esterified caprylic and capric acids were infused into a pig duodenum with severed pancreatic ducts. Despite a lack of pancreatic enzymes, non-esterified acids appeared in the portal vein following infusion. In the portal blood, $26 \%$ of the caprylic and $27 \%$ of the capric acids infused as MCT were recovered. This suggests that alternative pathways exist, distinct from those following hydrolytic activity of the pancreatic lipase. According to authors perhaps the MCT were intracellularly hydrolysed in enterocytes though the residual activity of gastric lipases could not be excluded. Such alternative pathways of hy- 
drolysis may be particularly important in the first weeks of life because, as Corring et al. (1978) found, a marked increase in lipase activity appears only after the third week of a piglet's life.

Due to their lower molecular weight and smaller size, MCFA are more soluble in water and biological liquids than long-chain fatty acids (LCFA). Whereas LCFA, the products of long-chain triacylglycerol digestion, are reassimilated into long-chain phospholipids and triacylglycerols, MCFA are absorbed directly into portal circulation and transported to the liver for rapid oxidation (Odle, 1997). This can provide the animal with readily available energy. According to Dicklin et al. (2006), the rate of MCT hydrolysis also depends on the chain length of the acid in question. When triacylglycerols of MCFA were given as separate substrates, lipase activity decreased progressively as fatty-acid chain length increased from $\mathrm{C} 4$ to $\mathrm{C} 10$. As combined substrates were used, tri-C6:0 was hydrolysed most rapidly, followed by $\mathrm{C} 4, \mathrm{C} 8$, and $\mathrm{C} 10$. The hydrolysis of $\mathrm{C} 6$ was 7 times greater than that of $\mathrm{C} 8$; therefore hexanoate may be preferentially utilised by piglets. Similar results were obtained in our experiment on piglets comparing the effect of $0.2 \%$ dietary supplements of caprylic and capric acids (Hanczakowska et al., 2011). Piglets receiving acids with shorter chain lengths (caprylic) grew faster than those receiving both of these acids or capric acid alone, though these differences were not statistically significant. All experimental animals grew significantly faster than controls (Table 1).

Table 1. The effect of MCFA ${ }^{1}$ or $\mathrm{MCT}^{2}$ supplement on piglet body weight gains (BWG)

\begin{tabular}{l|c|c|c|c}
\hline \multicolumn{1}{c|}{ Supplement } & $\begin{array}{c}\text { Dose } \\
\%\end{array}$ & $\begin{array}{c}\text { Experimental } \\
\text { days }\end{array}$ & $\begin{array}{c}\text { Improvement } \\
\text { of BWG (\%) }\end{array}$ & \multicolumn{1}{c}{ Authors } \\
\hline MCFA-C8 & 0.3 & $1-84$ & 18.7 & Hanczakowska et al., 2011 \\
MCFA-C10 & 0.3 & $1-84$ & 13.0 & Hanczakowska et al., 2011 \\
MCFA-C8+C10 & 0.3 & $1-84$ & 15.8 & Hanczakowska et al., 2011 \\
MCFA-C6+C8+C10+C12 & 0.2 & $1-42$ & 2.8 & Mohana Devi and Kim, 2014 \\
MCFA-C6+C10 & 0.3 & $28-58$ & 1.8 & Zentek et al., 2014 \\
MCT & 2.5 & $1-21$ & 11.0 & Decuypere and Dierick, 2003 \\
MCT & 1.2 & 28 & 0.4 & Lai et al., 2014 \\
MCT & 3.0 & 28 & 1.1 & Lai et al., 2014 \\
MCT & 4.8 & 28 & 2.0 & Lai et al., 2014 \\
MCT & 3.0 & $1-14$ & 7.4 & Yen et al., 2015 \\
\hline
\end{tabular}

${ }^{1}$ MCFA - Medium Chain Fatty Acid.

${ }^{2} \mathrm{MCT}$ - Medium Chain Triacylglycerol.

Price et al. (2013) found a higher digestibility in piglets of the MCT mixture containing mainly $\mathrm{C} 8$ and $\mathrm{C} 10$ than that of a long-chain fatty-acid triacylglycerol (LCT) mixture containing mainly palmitic and oleic acids (C16:0 and C18:1, respectively). On the other hand, piglets fed with LCT grew 22\% faster than those fed MCT. This difference was significant in the first week of the trial, but not in the second. Piglets more readily consumed feed containing LCT; according to the authors, this could be due to the organoleptic properties of or postabsorptive signals given by the MCT. Circulating fatty acids and ketone concentrations may induce feelings of satiety and thus reduce feed intake. Another possible reason for the reduced feed intake is the 
effect of MCT on ghrelin, a hormone produced by endocrine cells of the gastric mucosa; its main function is to control feed intake (Nakazato et al., 2001). Ghrelin may be acylated by MCFA and MCT which change its activity, but short-chain and long-chain fatty acids had no such effect (Nishi et al., 2005).

Lai et al. (2014) found slightly but not significantly better body weight gains in piglets after replacing a portion of the soybean oil in their diets with a mixture of MCT containing mainly caprylic acid (48\%) and similar amounts of caproic and capric acids (about 25\% each). Due to lower feed intake, the gain:feed ratio was significantly improved. A supplement of MCT also significantly improved the apparent digestibility of protein, which could be due to stimulation of cholecystokinin secretion by MCT in the small intestine of piglets (Stubbs and Stabile, 1985). In turn, cholecystokinin stimulates the secretion of pancreatic proteases, thus improving protein digestibility. Similar results (non-significant growth improvement and a significantly better gain: feed ratio) were obtained by Yen et al. (2015) using a supplement of MCTs with or without antibiotic (colistin sulphate). There was no interaction between these two supplements, which suggested different modes of action. Hong et al. (2012), using a mixture containing half-and-half caproic and caprylic MCT, found improved body weight gains in piglets when compared with a positive control (an antibiotic) in the first two weeks of the experiment only. In a later phase, the differences levelled off. A similar mixture of caprylic and capric acids was used by Li et al. (2015) to replace different amounts of soybean oil (from 14 to $28 \mathrm{~g}$; the control was $35 \mathrm{~g}$ of soybean oil). Piglet performance was similar: all experimental groups grew faster than controls during the first two weeks of the experiment, then the growth rate levelled off and there was no significant difference over the course of the experiment as a whole (days 1-28). According to the authors, this early improvement in performance could have been due to the above-mentioned traits of MCFA: better solubility in water and a shorter path of absorption and oxidation. Increasing the inclusion of MCT resulted in a linear decrease in feed intake. Results of supplementing piglet feed with MCFA or MCT obtained by different authors are summarised in Table 1.

In contrast to these generally positive results, Lee and Chiang (1994) found no improvement in growth or lower mortality rate in piglets fed MCT mixture containing $65 \%$ caprylic and $25 \%$ capric acids twice at 14 and $26 \mathrm{~h}$ of age and observed to weaning at 28 days of life. In any case, MCT had no adverse effect when given to pigs, or to rabbits and rats. Moreover, their safe use in human consumption to levels of $1 \mathrm{~g} / \mathrm{kg}$ has been confirmed in several clinical trials (Traul et al., 2000).

The amount of MCT used is also important. In the early experiment of Allee et al. (1972) higher dose (10\%) of MCT lowered piglet performance when compared to tallow, lard or coconut oil.

In the experiments described above, pure MCFA or MCT were used. In some experiments, MCFA were used in combination with other acids or microbial preparations to find possible synergism in their effect on pig performance.

Hanczakowska et al. (2013) supplemented piglet feed preserved with organic acids (propionic and fumaric) with caprylic and/or capric acids. Piglet body weight gains were numerically improved by MCFA compared to a diet with acidifiers and significantly compared to negative controls. There was no significant difference be- 
tween the negative controls and diets with organic acids only. This improvement was probably due to the better digestibility of protein and fat in piglets fed with MCFA (Table 2), which in turn could be the result of improvements in intestinal epithelial mucosal structure.

Table 2. Apparent digestibility of nutrients, \% (based on 4 litters per group) (Hanczakowska et al., 2013)

\begin{tabular}{lllll|l|l|l}
\hline \multicolumn{1}{c|}{ Item } & \multicolumn{1}{c|}{ Control } & \multicolumn{1}{c|}{$(\mathrm{PF})$} & $\left(\mathrm{PF}+\mathrm{C}_{8}\right)$ & $\left(\mathrm{PF}+\mathrm{C}_{10}\right)$ & $\left(\mathrm{PF}+\mathrm{C}_{8}+\mathrm{C}_{10}\right)$ & SEM \\
\hline Dry matter & 80.2 & 81.8 & 81.3 & 81.4 & 81.7 & 0.24 \\
Crude protein & $72.6 \mathrm{Aa}$ & $73.3 \mathrm{Aa}$ & $76.5 \mathrm{ABb}$ & $78.5 \mathrm{Bb}$ & $76.3 \mathrm{ABb}$ & 0.59 \\
Crude fat & $30.6 \mathrm{a}$ & $33.9 \mathrm{ab}$ & $31.03 \mathrm{a}$ & $39.9 \mathrm{~b}$ & $39.3 \mathrm{~b}$ & 1.23 \\
Crude fibre & $20.5 \mathrm{Aa}$ & $21.1 \mathrm{Aa}$ & $35.3 \mathrm{Bc}$ & $33.1 \mathrm{Bbc}$ & $28.4 \mathrm{ABb}$ & 1.58 \\
N-free extract & 90.7 & 91.3 & 90.9 & 90.8 & 91.4 & 0.30 \\
\hline
\end{tabular}

Mean values in the same row with different letters differ significantly; $\mathrm{P} \leq 0.01$ (A, B); $\mathrm{P} \leq 0.05$ (a, b, c).

$\mathrm{P}$ - propionic acid, $\mathrm{F}$ - fumaric acid.

Acid doses: $\mathrm{P}-0.25 \%, \mathrm{~F}-0.25 \%$, MCFA $-0.2 \%$. Piglet age 60 days.

In a similar experiment, Zentek et al. (2013), using fumaric and lactic acids with a supplement of MCFA, found no significant improvement in piglet performance.

A mixture of organic acids and MCFA was used as a replacer for a standard antibiotic supplement by Kuang et al. (2015). The control diet contained colistin sulphate, enramycin, and zinc oxide (one of the growth promoters which regulate intestinal microflora and decrease the release of histamine, thus preventing postweaning diarrhoea; however, it is toxic to pigs). The experimental supplement consisted of calcium formate, calcium lactate, citric acid, and MCFA. Piglets receiving acids were characterised by greater feed intake and body weight; greater apparent ileal digestibility of a majority of amino acids, including methionine, lysine, and threonine; and higher Ig-G concentration. Increased Lactobacillus content in the ileum was also observed.

Mohana Devi and Kim (2014), in a six-week experiment on piglets, used supplements of MCFA and/or the probiotic Enterococcus faecium. All experimental groups were characterised by growth rates superior to controls, but this difference was significant only between controls and groups receiving the probiotic. Hanczakowska et al. (2016), using similar diets, found significantly improved body weight of piglets at 70 days of age when capric acid was fed. A supplement of Enterococcus faecium improved body weight gains only in the last period of the experiment, i.e. between 56 and 70 days of age.

There are two possible reasons for these positive activities of MCFA: changes in intestinal microflora and improvement of intestinal mucosal structure.

\section{Antibacterial activity of MCFA}

The antimicrobial effect of organic acids is due mainly to their undissociated state (Eklund, 1983). As most MCFA are in undissociated, fat-soluble form, they can penetrate the semi-permeable membranes of bacteria and enter the cytoplasm. Inside the cell they dissociate due to an alkaline $\mathrm{pH}$; they lower $\mathrm{pH}$, which suppresses 
cytoplasmic enzymes and nutrient transport systems, leading to cellular death. In contrast to $E$. coli the lactobacilli, themselves acid producers, are acid resistant. In low concentration acids may be even utilized as nutrients (Hsiao and Siebert, 1999).

The bacteriostatic effect of saturated fatty acids (SFA) has been known for many years. In an experiment by Hassinen et al. (1951), the growth of bacterial cultures was inhibited by $\mathrm{C} 8$ and $\mathrm{C} 10$ though not significantly inhibited by $\mathrm{C} 4, \mathrm{C} 6, \mathrm{C} 16$, or C18 acids. According to Nieman (1954) C8-C14 acids are characterised by optimal inhibitory activity. In an experiment by Hanczakowska et al. (2013), short-chain fatty acids exerted a strong antibacterial effect on Clostridium perfringens in the piglet digestive tract. A supplement of MCFA reduced this improvement to the level of the control group. This may have been due to the high acidity of chyme. Marounek et al. (2003) found strong antibacterial activity on the part of MCFA at a $\mathrm{pH}$ about 6 or higher.

Intensive research on the antibacterial activity of MCFA began after the ban on the use of feed antibiotics. Early experiments were performed in vitro. Marounek et al. (2003) compared the antimicrobial activity of C2-C18 fatty acids in cultures of two strains of Escherichia coli grown on glucose. Neither short-chain fatty acids (C2-C6) nor fatty acids with long chains (C12-C18) influenced substrate utilisation. Only caprylic acid, and, to a lesser degree, capric acid, showed significant antimicrobial activity. Also, in an experiment by Skrivanová et al. (2006), medium-chain fatty acids proved to be more efficient antimicrobials than other, more polar organic acids tested. Transmission electron microscopy (TEM) revealed that caprylic and lauric acids damaged the cytoplasmic structure of bacteria; in some cases the separation of inner and outer membranes was apparent. Similar disruption of both the cell membranes and the cytoplasm of Clostridium cells under the influence of lauric acid were shown using TEM in an experiment by Shilling et al. (2013), who used virgin coconut oil (VCO) and its most active individual fatty acids. Lauric acid was the most inhibitory to Clostridium growth. Virgin coconut oil inhibited bacterial growth only when cells were exposed to lipolysed coconut oil.

Batovska et al. (2009) examined the antibacterial activity of MCFA and their 1-monoglycerides against several Gram-positive bacteria. They found that the monoglycerides were more active than the fatty acids, with monolaurin being the most active compound. Perhaps the glycerol moiety of monolaurin as a hydrophilic carrier transfers lauric acid through the bacterial membrane, where it exhibits antibacterial activity (Ruzin and Novick, 2000).

A synergistic relationship has been observed between monolaurin and monocaprin as well as between monolaurin and poorly active lauric acid. Also, Bergsson et al. (2001) found that monocaprin, especially at low concentrations, is more active than capric acid. Two-colour fluorescent and electron microscopy assays indicated that bacteria were killed by disintegration of the cell membrane, leaving the bacterial wall intact.

Dierick et al. (2003) used seeds of Cuphea, which earlier had been found to contain high amounts of MCFA (Graham and Knapp, 1989), supplemented with lipase, as a source of these acids in piglet nutrition. Enzymatically released MCFA (1.7 g per $\mathrm{kg}$ of digesta) tended to decrease the number of coliforms in the proximal small 
intestine, but increase it in the distal. No differences were noted for the total anaerobic microbial load in the gut. As can be seen from the results of Lai et al. (2014), lactic acid bacteria are the most sensitive to the antibacterial activity of MCT. There was no significant difference in the number of coliforms even at the highest doses of acids. Zentek et al. (2012) compared the effects of MCFA, uncoated and coated with vegetable fat and lecithin and added to weaned piglet feed. They found a rapid decrease in MCFA concentration along the gastrointestinal tract, regardless of encapsulation. An increase in the numbers of eubacteria, enterobacteriaceae, and two species of Lactobacillus in gastric content was also found. Changes in microflora under the influence of acids increased ammonia concentration in the distal small intestine. Yen et al. (2015) compared the effects of MCT mixture (C6:C8:C10 in the ratio 25:48:27) to those of an antibiotic (colistin sulphate). The MCT did not affect the microbial counts in the small intestine and caecum contents in piglets, though the coliform count in the colon decreased. The antibiotic decreased coliform counts in the caecum and colon, but not in the small intestine.

In an experiment by Hanczakowska et al. (2016), amounts of bacterial DNA were calculated in the jejunum and caecum (Table 3). Antimicrobial activity of MCFA is well documented. Number of two species of bacteria dangerous and common in piglet intestines i.e. Escherichia coli and Clostridium perfringens (Skrivanová et al., 2006) was determined in this experiment. Marounek et al. (2003) found that caprylic acid and to a lesser extent capric acid had a significant antimicrobial activity against $E$. coli which is consistent with the results of this experiment. In comparison to the control group, dietary caprylic acid significantly lowered the numbers of E. coli in both of these parts of the intestines, but the numbers of Clostridium were significantly reduced only in the caecum. Inhibitory effect of MCFA against Clostridium was found also in the experiment of Sprong et al. (2001).

Table 3. Microbial counts in small intestine (jejunum) and caecum digesta, $\log _{10} \mathrm{CFU} /(1 \mathrm{~g}$ chyme $)$ (based on 12 piglets per group) (Hanczakowska et al., 2016)

\begin{tabular}{|c|c|c|c|c|c|c|c|c|c|}
\hline & \multicolumn{3}{|c|}{$\begin{array}{l}\text { Experimental } \\
\text { group }(\mathrm{G})\end{array}$} & \multicolumn{2}{|c|}{$\begin{array}{c}\text { Supplemental } \\
\text { probiotic }(\mathrm{P})\end{array}$} & \multicolumn{3}{|c|}{$\mathrm{P}$-value } & \multirow[t]{2}{*}{ SEM } \\
\hline & $\mathrm{C}$ & $\mathrm{C} 8$ & $\mathrm{C} 10$ & 0 & + & G & $\mathrm{P}$ & GxP & \\
\hline \multicolumn{10}{|c|}{ Jejunum } \\
\hline E. coli & $3.12 \mathrm{~b}$ & $2.05 \mathrm{a}$ & $2.77 \mathrm{ab}$ & $2.99 \mathrm{~b}$ & $2.30 \mathrm{a}$ & 0.018 & 0.024 & $<0.001$ & 0.23 \\
\hline $\begin{array}{l}\text { Clostridium } \\
\text { perfringens }\end{array}$ & 3.31 & 2.84 & 3.13 & $3.71 \mathrm{~B}$ & $2.48 \mathrm{~A}$ & 0.208 & $<0.001$ & $<0.001$ & 0.17 \\
\hline \multicolumn{10}{|c|}{ Caecum } \\
\hline E. coli & $2.87 \mathrm{~b}$ & $2.18 \mathrm{a}$ & $2.35 \mathrm{ab}$ & 2.61 & 2.31 & 0.011 & 0.105 & $<0.001$ & 0.16 \\
\hline $\begin{array}{l}\text { Clostridium } \\
\text { perfringens }\end{array}$ & $3.41 \mathrm{~b}$ & $2.44 \mathrm{a}$ & $2.85 \mathrm{ab}$ & $3.76 \mathrm{~B}$ & $2.04 \mathrm{~A}$ & 0.026 & $<0.001$ & 0.221 & 0.22 \\
\hline
\end{tabular}

Mean values in the same row with different letters differ significantly at $\mathrm{P} \leq 0.01$ (A, B); $\mathrm{P} \leq 0.05$ (a, b). Acid doses: C8, C10 - 0.3\%; Supplement probiotic: Cylactin - 0.35x109 CFU per kg feed. Piglet age 60 days. 


\section{Effects of MCFA on intestinal epithelial mucosal structure}

The mucosal epithelium of the small intestine is the main site of nutrient degradation and absorption (Cera et al., 1988). The mucosa's functional surface area is increased by villi and crypts. The epithelial cells near the villus tip are the most mature and have the greatest digestive and absorptive capacity (Tang et al., 1999); therefore piglet weight gain is correlated with villus height (Zijlstra et al., 1996).

This relationship was found by Dierick et al. (2003) in the above-mentioned experiment with Cuphea seeds with a lipase supplement as a source of MCFA in diets for piglets. The results indicated a trend towards improved performance parameters, as well as significantly greater villus height and lesser crypt depth. In our earlier experiment (Hanczakowska et al., 2011), both caprylic and capric acids, fed separately or together, significantly improved piglet body weight gain and resulted in greater villus height, but compared to the control group, the latter difference was significant only in the case of capric acid.

Table 4. Mucosal epithelial structure of the ileum (based on 6 piglets per group) (Hanczakowska et al. 2011)

\begin{tabular}{l|c|c|c|c|c}
\hline \multicolumn{1}{c}{ Ileum morphology } & \multicolumn{1}{c|}{ Control } & \multicolumn{1}{c|}{$\mathrm{C}_{8}$} & $\mathrm{C}_{10}$ & $\mathrm{C}_{8+\mathrm{C} 10}$ & $\mathrm{SEM}$ \\
\hline Villus height $(\mu \mathrm{m})$ & $233 \mathrm{~A}$ & $267 \mathrm{AB}$ & $306 \mathrm{~B}$ & $268 \mathrm{AB}$ & 7.86 \\
Villus width $(\mu \mathrm{m})$ & 116 & 114 & 120 & 116 & 2.25 \\
Crypt depth $(\mu \mathrm{m})$ & $280 \mathrm{a}$ & $304 \mathrm{ab}$ & $338 \mathrm{~b}$ & $280 \mathrm{a}$ & 0.02 \\
Villus height/crypt depth & 0.835 & 0.959 & 0.908 & 0.971 & 0.03 \\
\hline
\end{tabular}

Mean values in the same row with different letters differ significantly: $\mathrm{P} \leq 0.01$ (A, B); $\mathrm{P} \leq 0.05$ (a, b).

MCFA $0.3 \%$. Piglet age 60 days.

The effect of a mixture of short-chain organic acids (fumaric and lactic), alone or supplemented with MCFA (caprylic and capric acids), on gut morphology and the local intestinal immune system in weaned piglets was studied by Ferrara et al. (2016), who found a trend (not significant) towards longer villi in the mid-jejunum of piglets fed with the MCFA supplement. Short-chain organic acids slightly increased the number of intra-epithelial lymphocytes in the jejunal epithelium; thus they might have a beneficial effect on local immunity. MCFA had no effect on lymphocyte numbers.

\section{Conclusions}

Generally, medium-chain fatty acids and their triacylglycerols added to piglet diets in moderate amount (MCFA $0.2-0.3 \%$, MCT $1.2-4.8 \%$ ) are a good source of energy for cells of the intestinal epithelium. They improve piglet performance, due mainly to the short pathway of their absorption and oxidation, as well as exerting an antibacterial effect and improving intestinal epithelial structure.

\section{References}

Alle e G.L., Roms os D.R., L eveille G.A., B a ker D.H. (1972). Metabolic consequences of dietary medium-chain triglycerides in the pig. Proc. Soc. Exp. Biol. Med., 139: 422-428. 
A nadón A. (2006). The EU ban of antibiotics as feed additives: alternatives and consumer safety. J. Vet. Pharm. Therap., 29: 41-44.

B atovska D.I., Todorova I.T., Tsvetkova I.V., Najdenski H.M. (2009). Antibacterial study of the medium chain fatty acids and their 1-monoglycerides: individual effects and synergistic relationship. Pol. J. Microbiol., 58: 43-47.

B a y l e y H.S., H o $1 \mathrm{~m}$ e s H.G., S t e v e n s o n K.R. (1974). Digestion by the pig the energy and nitrogen in dried, ensiled and organic-acid-preserved corn: with observations on the starch content of digesta samples. Can. J. Anim. Sci., 54: 377-383.

B ergs son G., Arnfinns s on J., S teingrims s o n O., Thormar H. (2001). Killing of grampositive cocci by fatty acids and monoglycerides. APMIS, 109: 670-678.

Cer a K.R., Mahan D.C., Cross R.F., Re inhart G.A., Whit moyer R.E. (1988). Effect of age, weaning and postweaning diet on small intestinal growth and jejunal morphology in young swine. J. Anim. Sci., 66: 574-584.

Corring T., A u maitre A., Durand G. (1978). Development of digestive enzymes in the piglet from birth to 8 weeks. I. Pancreas and pancreatic enzymes. Nutr. Metab., 22: 231-243.

D e c u y p e r e J.A., D i e r i c k N.A. (2003). The combined use of triacylglycerols containing mediumchain fatty acids and exogenous lipolytic enzymes as an alternative to in-feed antibiotics in piglets: concept, possibilities and limitations. An overview. Nutr. Res. Rev., 16: 193-209.

D i ckl in M.E., R ob in s on J.L., L i n X., O d l e J. (2006). Ontogeny and chain-length specificity of gastrointestinal lipases affect medium-chain triacylglycerol utilization by newborn pigs. J. Anim. Sci., 84: 818-825.

D i e r i c k N.A., D e c u y p e r e J.A., D e g e y t e r I. (2003). The combined use of whole Cuphea seeds containing medium chain fatty acid and an exogenous lipase in piglet nutrition. Arch. Tierernähr., 57: 49-63.

E k l u n d T. (1983). The antimicrobial effect of dissociated and undissociated sorbic acid at different $\mathrm{pH}$ levels. J. Appl. Bacteriol., 54: 383-389.

F errara F., Ted in L., P i e per R., M e yer W., Z e n tek J. (2016). Influence of medium-chain fatty acids and short-chain organic acids on jejunal morphology and intra-epithelial immune cells in weaned piglets. J. Anim. Physiol. Anim. Nutr., Doi: 10.1111/jpn.12490, 10 pp.

Gi esting D.W., E a s te r R.A. (1985). Response of starter pigs to supplementation of corn-soybean meal diets with organic acids. J. Anim. Sci., 60: 1288-1294.

Grah a m S.A., Kn a p p S.J. (1989). Cuphea: a new plant source of medium-chain fatty acids. Crit. Rev. Food Sci. Nutr., 28: 139-173.

Guill ot E., L e m a r c h a 1 P., D h or n e T., R e r a t A. (1994). Intestinal absorption of medium chain fatty acids: in vivo studies in pigs devoid of exocrine pancreatic secretion. Br. J. Nutr., 72: 545-553.

H a n c z a k o w s k a E., S z e w c z y k A., O k oń K. (2011). Effect of dietary caprylic and capric acids on piglet performance and mucosal epithelium structure of ileum. J. Anim. Feed Sci., 20: 545-554.

Hanczakowska E., Szew czyk A., Św i ątki ew icz M., Okoń K. (2013). Short- and medium-chain fatty acids as a feed supplement for weaning and nursery pigs. Pol. J. Vet. Sci., 16: $647-654$.

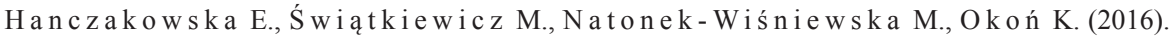
Medium chain fatty acids (MCFA) and/or probiotic Enterococcus faecium as a feed supplement for piglets. Livest. Sci., 192: 1-7.

H a s s in e n J.B., D u r b in G.T., B e r n h a r t F.W. (1951). The bacteriostatic effects of saturated fatty acids. Arch. Biochem. Biophys., 31: 183-189.

H e o K.N., L in X., H a n I.K., O d l e J. (2002). Medium-chain fatty acids but not l-carnitine accelerate the kinetics of $\left[{ }^{14} \mathrm{C}\right]$ triacylglycerol utilization by colostrum-deprived newborn pigs. J. Nutr., 132: 1989-1994.

H o n g S.M., H w ang J.H., K i m I.H. (2012). Effect of medium-chain triglyceride (MCT) on growth performance, nutrient digestibility, blood characteristics in weanling pigs. Asian-Aust. J. Anim. Sci., 25: 1003-1008.

H s i a o C.P., S i e b e r t K.J. (1999). Modeling the inhibitory effects of organic acids on bacteria. Int. J. Food Microbiol., 47: 189-201.

Kuang Y., Wang Y., Zhang Y., Song Y., Zhang X., Lin Y., Che L., Xu S., Wu D., $\mathrm{X}$ u e B., F a n g Z. (2015). Effect of dietary combination of organic acids and medium chain fatty 
acids as a replacement of zinc oxide on growth, digestibility and immunity of weaned pigs. Anim. Feed Sci. Technol., 208: 145-157.

L a i W.K., Yen H.C., L in C.S., Chiang S.H. (2014). The effects of dietary medium-chain triacylglycerols on growth performance and intestinal microflora in young pigs. J. Anim. Feed Sci., 23: 331-336.

L e e H.F., Chiang S.H. (1994). Energy value of medium-chain triglycerides and their efficacy in improving survival of neonatal pigs. J. Anim. Sci., 72: 133-138.

Li Y., Zhang H., Yang L., Wang T. (2015). Effect of medium-chain triglycerides on growth performance, nutrient digestibility, plasma metabolites and antioxidant capacity in weanling pigs. Anim. Nutr., 1: 12-18.

L o h T.C., F o o H.L., N g u y e n T.T., C h o e D.W. (2013). Growth performance, plasma fatty acids, villous height and crypt depth of preweaning piglets fed with medium chain triacylglycerol. Asian Australas. J. Anim. Sci., 26: 700-704.

L u J.J., Z o u X.T., W a n g Y.M. (2008). Effects of sodium butyrate on the growth performance, intestinal microflora and morphology of weanling pigs. J. Anim. Feed Sci., 17: 568-578.

Marounek M., Skrivanová E., Rada V. (2003). Susceptibility of Escherichia coli to C2-C18 fatty acids. Folia Microbiol. (Praha), 48: 731-735.

M o ha na D e vi S., K i m I.H. (2014). Effect of medium chain fatty acids (MUFA) and probiotic (Enterococcus faecium) supplementation on the growth performance, digestibility, and blood profiles in weanling pigs. Vet. Med., 59: 527-535.

Nakazato M., Murakami N., Date Y., Kojami M., Matsuo H., Kangawa K., Mats u k u r a S. (2001). A role of ghrelin in the central regulation of feeding. Nature, 409: 194-198.

$\mathrm{N}$ i e $\mathrm{m}$ a $\mathrm{n} \mathrm{C}$. (1954). Influence of trace amounts of fatty acids on the growth of microorganisms. Bacteriological Rev., 18: 147-163.

Nishi Y., Hiejima H., Hos oda H., Kaiya H., Mori K., Fukue Y., Yanas e T., Nawat a H., Kangaw a K., K oj im a M. (2005). Ingested medium-chain fatty acids are directly utilized for the acyl modification of ghrelin. Endocrinology, 146: 2255-2264.

O d l e J. (1997). New insights into the utilization of medium-chain triglycerides by the neonate: observations from a piglet model. J. Nutr., 127: 1061-1067.

O p re a n L., I a n c u R., S t a n R., T r a ş c ă C. (2011). Comparison between types of feeding on goat milk composition. Anim. Sci. Biotechnol., 44: 76-79.

P a r t a n e n K., M r o z Z. (1999). Organic acids for performance enhancement in pig diets. Nutr. Res. Rev., 12: 117-145.

Piva A., Morlacchini M., Cas adei G., Gatta P.P., Biagi G., Prandini A. (2002). Sodium butyrate improves growth performance of weaned piglets during the first period after weaning. Ital. J. Anim. Sci., 1: 35-41.

P l a y o u s t M.R., I s s e $1 \mathrm{~b}$ a c h e r K.J. (1964). Studies on the intestinal absorption and intramucosal lipolysis of a medium-chain triglyceride. J. Clin. Invest., 43: 878-885.

Price K.L., Lin X., van Heugten E., Odle R., Willis G., Odle J. (2013). Diet physical form, fatty acid chain length, and emulsification alter fat utilization and growth of newly weaned pigs. J. Anim. Sci., 91: 783-792.

R u z i n A., N o v i c k R.P. (2000). Equivalence of lauric acid and glycerol monolaurate as inhibitors of signal transduction in Staphylococous aureus. J. Bacteriol., 182: 2668-2671.

Shilling M., Mat t L., R ub in E., Vis it a c i on M.P., Halle r N.A., Grey S.F., Woolvert o n C.J. (2013). Antimicrobial effects of virgin coconut oil and its medium-chain fatty acids on Clostridium difficile. J. Med. Food, 16: 1079-1085.

S k rivanová E., M a r o u n e k M., B e n d a V., B re z i n a P. (2006). Susceptibility of Escherichia coli, Salmonella sp. and Clostridium perfringens to organic acids and monolaurin. Vet. Med., 51: 81-88.

Skrivanová E., Molatová Z., Skrivanová V., Maroun e k M. (2009). Inhibitory activity of rabbit milk and medium-chain fatty acids against enteropathogenic Escherichia coli O128. Vet. Microbiol., 135: 358-362.

S prong R.C., Huls te in M.F.E., Van der M e e r R. (2001). Bactericidal activities of milk lipids. Antimicrobial Agents Chemiotherapy, 45: 1298-1301.

S t u b b s R.S., S t a bi l e B.E. (1985). Role of cystokinin in pancreatic response to intraluminal amino acids and fat. Amer. J. Physiol., 248: G347-G352. 
S u iry an ra y n a M.V.A.N., R a man a J.V. (2015). A review of the effects of dietary organic acids fed to swine. J. Anim. Sci. Biotechnol., 6, 45, DOI 10.1186/s40104-015-0042-z

Tang M., La arveld B., Van Kessel A.G., Hamilton D.L., Estrada A., P a ti en ce J.F. (1999). Effect of segregated early weaning on postweaning small intestinal development in pigs. J. Anim. Sci., 77: 3191-3200.

Traul K.A., Drie d ger A., Ing le D.L., N a khas i D. (2000). Review of the toxicologic properties of medium-chain triglycerides. Food. Chem.Toxicol., 38: 79-98.

W a 1 z O.P., P a l a u f J. (1997). Retention and utilization of amino acids in piglets fed ad libitum or restrictively diets supplemented with organic acids. Arch. Tierernähr., 50: 227-238.

Yen H-C., L a i W-K., L in C-S., C h i an g S-H. (2015). Medium-chain triglyceride as an alternative of in-feed colistin sulfate to improve growth performance and intestinal microbial environment in newly weaned pigs. Anim. Sci. J., 86: 99-104.

You n g L.G., B rown R.G., S h a r p B.A. (1970). Propionic acid preservation of corn for pigs. Can. J. Anim. Sci., 50: 711-715.

Zentek J., Buchheit-Renko S., Männer K., Pieper R., Vahjen W. (2012). Intestinal concentrations of free and encapsulated dietary medium-chain fatty acids and effects on gastric microbial ecology and bacterial metabolic products in the digestive tract of piglets. Arch. Anim. Nutr., 66: 14-26.

Zentek J., Ferrara F., Pieper R., Tedin L., Meyer W., Vahjen W. (2013). Effects of dietary combination of organic acids and medium chain fatty acids on the gastrointestinal microbial ecology and bacterial metabolites in the digestive tract of weaning piglets. J. Anim. Sci., 91: 3200-3210.

Zijlstra R.T., Whang K-Y., E a ster R.A., O d l e J. (1996). Effect of feeding a milk replacer to early-weaned pigs on growth, body composition, and small intestinal morphology, compared with suckled littermates. J. Anim. Sci., 74: 2948-2959.

Received: 8 XI 2016

Accepted: 2 III 2017 